\title{
DRUSAS DEL NERVIO ÓPTICO Y DEFECTOS CAMPIMÉTRICOS SEVEROS
}

\section{OPTIC NERVE DRUSEN AND DEEP VISUAL FIELDS DEFECTS}

\author{
CALVO-GONZÁLEZ C ${ }^{1}$, SANTOS-BUESO E ${ }^{2}$, DÍAZ-VALLE D ${ }^{2}$, RECHE-FRUTOS ${ }^{1}$, \\ MORICHE-CARRETERO $\mathrm{M}^{3}$, BENÍTEZ-DEL-CASTILLO JM ${ }^{2}$, GARCÍA-SÁNCHEZ J²
}

\begin{abstract}
RESUMEN
Objetivo: Las drusas del nervio óptico deben ser incluidas en el diagnóstico diferencial de pseudopapiledema, ya que pueden resultar difícilmente identificables mediante funduscopia. Por ello se hace necesaria la utilización de la ecografía, como gold standard en su diagnóstico. Se ha correlacionado la severidad de las drusas papilares con la reducción del espesor de la capa de fibras nerviosas medidas por tomografía óptica de coherencia y con la existencia de defectos importantes en el campo visual. Demostrar la existencia de una alteración campimétrica asociada a la presencia de drusas del nervio óptico extensas.

Métodos: Estudio descriptivo prospectivo de campimetrías realizadas por una serie de 5 pacientes afectos de drusas del nervio óptico severas diagnosticadas mediante ultrasonografía.

Resultados: Se han observado defectos campimétricos destacables de diversa severidad en todos los pacientes estudiados, que oscilan entre escalón nasal y defectos de hemicampos severos.
\end{abstract}

\begin{abstract}
Purpose: Optic nerve drusen needs to be included in the differential diagnosis of pseudopapilledema. As the identification of this entity by funduscopy may be difficult, ultrasonography has thus become the gold standard for its diagnosis. Severe optic nerve drusen has been correlated with a reduction of the nerve fiber layer measured by optic coherence tomography and with the presence of serious visual field defects. To demonstrate the relationship between extensive optic nerve drusen and visual field defects.

Method: A prospective observational study of the visual fields of a series of 5 patients with severe optic nerve drusen diagnosed by ultrasonography.

Results: Visual field defects of widely differing severity, from inferior nasal quadrant to severe hemivisual field defects, were described in each patient studied.

Conclusions: Visual field defects of diverse severity are common in patients with deep optic nerve drusen. For that reason ultrasonography and/or opti-
\end{abstract}

Recibido: 7/9/05. Aceptado: 19/5/06.

Unidad de Superficie e Inflamación Ocular. Servicio de Oftalmología. Hospital Clínico San Carlos. Madrid. España.

1 Licenciado en Medicina.

2 Doctor en Medicina.

3 Doctor en Medicina. Hospital Universitario La Paz. Madrid. España.

Correspondencia:

C. Calvo González

Servicio de Oftalmología. Hospital Clínico San Carlos

Avda. Prof. Martín Lagos, s/n

28040 Madrid

España

E-mail: calvo_glez@yahoo.es 
Conclusiones: Los pacientes afectos de drusas papilares profundas presentan con frecuencia alteraciones en su campo visual de profundidad variable, por lo que los autores recomiendan un seguimiento campimétrico y/o con tomografía de coherencia óptica en estos casos.

Palabras clave: Drusas nervio óptico, defectos campimétricos, ecografía. cal coherence tomography is highly recommended where such visual field defects exist (Arch Soc Esp Oftalmol 2006; 81: 269-274).

Key words: Optic nerve drusen, campimetric defects, sonography.

\section{INTRODUCCIÓN}

Las drusas del nervio óptico (DNO) constituyen formaciones globulosas hialinas de 5 a 1.000 micras de diámetro, comúnmente situadas por delante de la lámina cribosa. Contienen aminoácidos, ácidos ribonucleicos, una pequeña cantidad de hierro y calcio, lo que determina que sean estructuras parcialmente calcificadas. Aunque su patogénesis permanece desconocida, las teorías más aceptadas sugieren una alteración en el flujo axoplásmico de las células ganglionares. Adicionalmente, los canales esclerales pequeños parecen asociarse con más frecuencia a la presencia de DNO. Clínicamente, se ha descrito una incidencia de 3.4 por cada 1.000 adultos. No obstante, los estudios histológicos sobre autopsia de cadáver reflejan una incidencia mayor. Suelen ser bilaterales y asimétricas, con una mayor predilección por el sexo femenino. Las DNO se asocian con relativa frecuencia a anomalías vasculares oculares, tales como, presencia de arterias ciliorretinianas, tortuosidad pronunciada, bifurcaciones anormales, colaterales retino-coroideas, hemorragias, neovascularización peripapilar y enfermedades vasculares oclusivas, entre otras (1). Las DNO pueden dar lugar a una elevación anómala de la papila, siendo necesario establecer el diagnóstico diferencial con el papiledema.

Clínicamente pueden disminuir la agudeza visual central (poco frecuente) así como producir defectos campimétricos, algunos simulando un patrón glaucomatoso. Este hallazgo, unido a una difícil interpretación del disco óptico por la presencia de las drusas, aumenta la complejidad en el diagnóstico y seguimiento de pacientes con posibles lesiones glaucomatosas. Asimismo, se ha correlacionado la severidad de la DNO clínicamente visibles con la reducción del espesor de la capa de fibras nerviosas medidas por tomografía de coherencia óptica
(OCT) y con los defectos de campo visual (2). El diagnóstico más fiable de las DNO lo proporciona la ecografía en modo $\mathrm{B}$, aunque también pueden detectarse mediante angiografía fluoresceínica, tomografía axial computarizada (TAC), métodos electrofisiológicos $\mathrm{y}$, las drusas visibles, por funduscopia.

Presentamos 5 casos clínicos que ponen de manifiesto la existencia de defectos de campo visual profundos, permanentes y con patrón glaucomatoso en pacientes con DNO severas, así como la utilidad de la ecografía y de la fotografía de alta definición en el diagnóstico diferencial de la papila elevada.

\section{SUJETOS, MATERIAL Y MÉTODOS}

Se realiza un estudio prospectivo de cinco pacientes (10 ojos) diagnosticados de DNO. Los criterios de inclusión son: 1) diagnóstico de DNO obtenido mediante funduscopia y/o ultrasonografía modo B, 2) drusas visibles severas y 3 ) alta fiabilidad obtenida en pruebas perimétricas. Los criterios de exclusión incluyen: 1) hipertensión ocular o glaucoma previamente diagnosticado, 2) evidencia de otras enfermedades oculares y 3) presencia de patología macular secundaria a alteraciones relacionadas con las drusas u otras causas de déficit visual.

Ecográficamente (ultrasonidos modo-B), las DNO se presentan como estructuras redondeadas hiperreflectantes con sombra acústica en ganancias medias. Se realizan fotografías de alta definición mediante retinógrafo no midriático (CR-DGi de Canon) y ecografías modo B (Ecógrafo Toshiba Power Vision 6000). En todos los casos se han realizado campimetrías computerizadas mediante el programa 24.2 del perímetro Humphrey FA II (Carl Zeiss, Inc, San Leandro, CA, EEUU), hasta conseguir campimetrías fiables, con una proporción infe- 
rior al $20 \%$ de falsos negativos y falsos positivos. Se han recogido los datos relativos a la máxima agudeza visual corregida (MAVC) en cada ojo, defecto medio (en $\mathrm{dB}$ ) y patrón de afectación campimétrica.

\section{RESULTADOS}

Se han estudiado 10 ojos de 5 pacientes, de los cuales cuatro son hombres. La edad media es de 57,6 (rango, 42-78 años). La agudeza visual mejor corregida media es de 0,78 , destacando una MAVC $\geq 0,5$ (logMAR) en todos ellos. La totalidad de los casos (10 ojos) presentaban DNO severas, cuyo diagnóstico se ha confirmado mediante la detección de una imagen hiperintensa en la cabeza del NO mediante ecografía modo B (figs. 1 y 2 ).

Se analizan las perimetrías de alta fiabilidad obtenidas mediante realización de Humphrey 24.2, observando una afectación del campo visual (CV) en el $100 \%$ de los casos. El defecto medio oscila entre $-2,39$ y $-22,7(\mathrm{~dB})$, con un valor medio calculado de $-9,8$. Las alteraciones campimétricas encontradas son las siguientes, enumeradas en orden decreciente de frecuencia: $40 \%$ de escotoma arciforme inferior, de los que un $50 \%$ presentan adi- cionalmente escalón nasal, $40 \%$ afectación de hemicampo inferior (50\% con afectación leve de hemicampo superior), $10 \%$ de arciforme superior y escalón nasal y $10 \%$ con escalón nasal aislados (figs. 1 y 2).

Los resultados obtenidos se presentan en la tabla I.

\section{DISCUSIÓN}

Las DNO superficiales suelen ser fácilmente identificables con las técnicas habituales de diagnóstico. Sin embargo, en el caso de las DNO profundas y enterradas puede resultar más compleja su identificación. Esta situación se describe con más frecuencia en la infancia, aunque existe una fuerte evidencia de que, con el paso de los años, se produce un aumento lento y progresivo en el tamaño y la visibilidad de las mismas (1). La difícil visualización de las drusas enterradas hace imprescindible su correcta diferenciación de un papiledema verdadero, haciéndose necesarios métodos diagnósticos complementarios que apoyen la presencia de este tipo de drusas. Existen distintas pruebas para determinar la presencia de drusas en la cabeza del nervio óptico (NO). La más sencilla y económica es la fun-
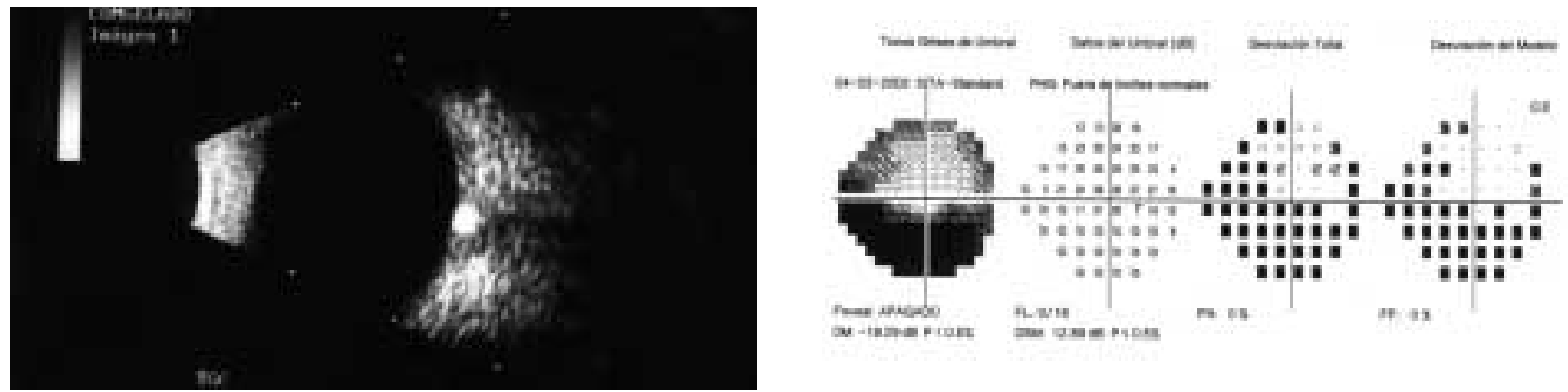

Fig. 1: Ecografía modo B y campimetría del ojo derecho del paciente número 3 con drusas profundas y enterradas en la cabeza del nervio óptico.
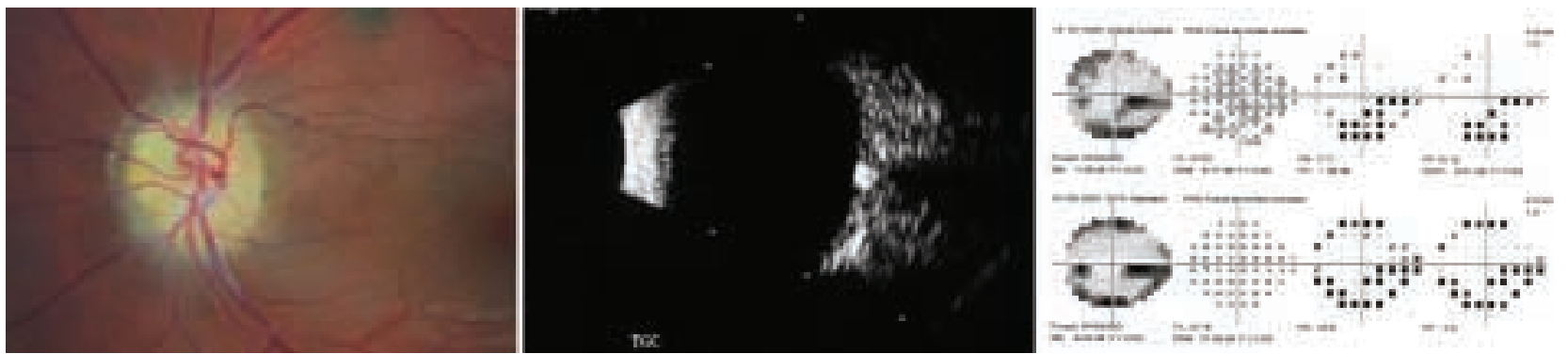

Fig. 2: Funduscopia, ecografía modo B y campimetrías seriadas en DNO. 
Tabla I. Resultados

\begin{tabular}{llll}
\hline $\begin{array}{l}\text { Paciente } \\
\text { (sexo, edad) }\end{array}$ & Ojo, MAVC* & DM† $(\mathrm{dB})$ & Defecto CV \\
\hline 1, hombre, 49 & OD: 0,9 & $-2,39$ & Arciforme inferior leve \\
1, hombre, 49 & OI: 0,8 & -8 & Arciforme inferior, amenaza foveal \\
2, hombre, 57 & OD: 1 & $-4,3$ & Arciforme superior, escalón nasal \\
2, hombre, 57 & OI: 1 & $-6,1$ & Arciforme inferior, escalón nasal \\
3, hombre, 72 & OD: 0,6 & $-18,3$ & Extenso hemicampo inferior \\
3, hombre, 72 & OI: 0,5 & $-22,7$ & Extenso en hemicampos \\
4, hombre, 78 & OD: 0,5 & $-17,20$ & Extenso en hemicampos \\
4, hombre, 78 & OI: 0,6 & $-12,09$ & Hemicampo inferior \\
5, mujer, 42 & OD: 1 & $-3,1$ & Escalón nasal \\
5, mujer, 42 & OI: 1 & $-4,5$ & Escalón nasal, arciforme inferior \\
\hline \hline
\end{tabular}

*MAVC: agudeza visual máxima corregida; $\mathrm{DM} \dagger$ : defecto medio $(\mathrm{dB})$.

duscopia, aunque presenta una importante limitación en la identificación de depósitos profundos o enterrados. La TAC resulta útil por su capacidad de mostrar el componente cálcico de las drusas como puntos brillantes identificables. Su coste elevado, así como la incapacidad de realizar cortes suficientemente finos en la región de la cabeza del NO, hacen de la TAC una prueba no adecuada como diagnóstico de rutina. Asimismo, resulta útil la angiografía fluoresceínica. La autofluorescencia de las DNO previa a la inyección de contraste resulta característica, así como la presencia de hiperfluorescencia en el paso tardío del mismo (1). No obstante, la prueba de elección en el diagnóstico de las DNO es la ecografía en modo B. Es un método con gran sensibilidad y una rentabilidad diagnóstica mayor que la detección de la autofluorescencia preinyección o el TAC orbitario (3).

Desde el punto de vista clínico, la disminución de la agudeza visual debida a la presencia de drusas es infrecuente. Por el contrario, los defectos de campo visual se describen comúnmente asociados a las DNO. Diversos estudios han demostrado una mayor frecuencia y severidad de alteraciones perimétricas en pacientes con DNO visibles, así como edad más avanzada (1-5), siendo los defectos campimétricos menos frecuentes en pacientes con DNO enterradas (6). Aunque las pérdidas más dramáticas de CV en pacientes con drusas suelen relacionarse con complicaciones vasculares asociadas, las drusas pueden ser asimismo responsables del defecto. En estos casos pueden encontrarse alteraciones pupilares del tipo defecto pupilar aferente relativo.

Las alteraciones más comúnmente observadas, descritas como lentamente progresivas, son defectos arciformes, sobre todo inferiores, constricción generalizada y agrandamiento de la mancha ciega. La pérdida del campo visual puede corresponder directamente o no a la localización de las DNO. Los mecanismos fisiopatogénicos son varios: compromiso del transporte axonal en un ojo con canal escleral pequeño con desgaste gradual secundario de las fibras del NO, compresión directa de las fibras prelaminares por las drusas e isquemia en la cabeza del NO (1). En nuestra serie hemos observado defectos campimétricos de profundidad variable, que muestran patrones similares a los descritos en la literatura. Por otra parte, los defectos más acusados se han detectado en pacientes con drusas más voluminosas en las imágenes ecográficas.

Mediante determinación con OCT y polarimetría láser de barrido (GDx) se ha descrito una disminución en la capa de fibras nerviosas en pacientes con DNO, mayor pérdida en relación con un mayor número y visibilidad de las drusas. La OCT parece ser un indicador más sensible y precoz para detectar la pérdida de fibras nerviosas comparado con la perimetría y la fotografía de la capa de fibras $(2,7)$. Los autores recomiendan realizar ecografía modo B para confirmar o descartar la existencia de DNO así como realizar un seguimiento campimétrico y/o con OCT para monitorizar la afectación de la capa de fibras nerviosas en estos pacientes.

\section{BIBLIOGRAFÍA}

1. Auw-Haedrich C, Staubach F, Witschel H. Optic disk drusen. Surv Ophthalmol 2002; 47: 515-532.

2. Roh S, Noecker RJ, Schuman JS, Hedges TR 3rd, Weiter $J J$, Mattox C. Effect of optic nerve head drusen on nerve fiber layer thickness. Ophthalmology 1998; 105: 878-885.

3. Kurz-Levin MM, Landau K. A comparison of imaging 
techniques for diagnosing drusen of the optic nerve head. Arch Ophthalmol 1999; 117: 1045-1049.

4. Wilkins JM, Pomeranz HD. Visual manifestations of visible and buried optic disc drusen. J Neuroophthalmol 2004; 24: 125-129.

5. Lee AG, Zimmerman MB. The rate of visual field loss in optic nerve head drusen. Am J Ophthalmol 2005; 139: 1062-1066.
6. Katz BJ, Pomeranz HD. Visual field defects and retinal nerve fiber layer defects in eyes with buried optic nerve drusen. Am J Ophthalmol 2006; 141: 248-253.

7. Tatlipinar S, Kadayifcilar S, Bozkurt B, Gedik S, Karaagaoglu E, Orhan M, et al. Polarimetric nerve fiber analysis in patients with visible optic nerve head drusen. J Neuroophthalmol 2001; 21: 245-249. 